\title{
O.S.K.A.R.- Fremdsprachentheater spielt Zeitumwandlung
}

\section{Frank Fischäss}

\begin{abstract}
Zusammenfassung
Aus der systematischen Verzahnung szenischer Interpretationen von Texten, Bildern und Themen resultiert Fremdsprachentheater. Zeitumwandlung repräsentiert den Titel eines Theaterstücks, das im Frühjahr 2006 am University College Cork von Studierenden entwickelt und aufgeführt wurde. Literarische Texte sowie Texte, die von den Studierenden selbst verfasst wurden, bilden das Grundmaterial für die Erarbeitung von Szenen, die sich in eine vorgegebene Handlungsstruktur einfügen. Die Konzeption von Zeitumwandlung verfolgt das Ziel, neben der Erweiterung theoretischer Kenntnisse in der Fremdsprache auch Lernfortschritte der Kursteilnehmer im Sprachhandeln und in der szenischen Interpretation literarischer Texte zu erzielen. Um eine Vorstellung von der Arbeitsweise im Rahmen der Kursarbeit zu vermitteln, werden exemplarisch Arbeitsanweisungen und -einheiten beschrieben. Abschließend werden erzielte Arbeitsergebnisse und auch Möglichkeiten zur weiteren Umsetzung dieses ganzheitlichen Unterrichtskonzepts dargestellt.
\end{abstract}

\section{Einführung}

Zeitumwandlung ist der Titel eines Theaterstücks, das im Frühjahr 2006 am University College Cork (UCC) erstmals von Germanistik-Studierenden geschrieben und aufgeführt wurde. Im Folgenden wird die Erarbeitung der im Montagestil zusammengefügten Szeneninhalte vorgestellt. Die Konzeption von Zeitumwandlung verfolgt das Ziel, neben der Erweiterung theoretischer Kenntnisse in der Fremdsprache auch Lernfortschritte der Kursteilnehmer im Sprachhandeln zu erzielen. Mit Hilfe eingestreuter Arbeitsanweisungen und ausgewählter Unterrichtsschritte soll eine Vorstellung von der Arbeitsweise im Rahmen der Kursarbeit vermittelt werden. In der Schlussreflexion werden die erzielten Arbeitsergebnisse bewertet und Möglichkeiten zur weiteren Umsetzung dieses ganzheitlichen Unterrichtskonzepts erwogen. 


\section{Das Projekt Zeitumwandlung: Voraussetzungen und Rahmenbedingungen}

\subsection{O.S.K.A.R. - Fremdsprachentheater}

Zur Umsetzung ganzheitlich und ästhetisch ausgerichteter Lehrverfahren im dramapädagogischen Fremdsprachenunterricht wurde die Projektidee Ö.S.K.A.R. - Fremdsprachentheater"geboren:
$\mathbf{O}$ riginalität einsetzen
O ffen meinen
O ptionen wahrnehmen
$\mathbf{S}$ ituationen schaffen
S zenarien diskutieren
S prachkenntnisse verbessern
K onzeption
umsetzen
$\mathbf{K}$ reativität ausleben
$\mathbf{K}$ onversation erleben
A uthentisch als
A kteur in
A ktion spielen
R ollen schreiben
$\mathbf{R}$ esultate genießen
$\mathbf{R}$ eaktionen erproben

Es ging in diesem Projekt um die schrittweise Erarbeitung eines Theaterstücks durch die Studierenden. Durch die Montage einzelner szenischer Interpretationen von Themen, Texten und Bildern entsteht ein in sich geschlossenes Stück, dessen Szenen von den Kursteilnehmern (KT) sprachlich entwickelt, geschrieben und aufgeführt werden.

Der Kursleiter (KL) konnte schon in Kanada Erfahrungen mit Fremdsprachentheater sammeln. In der Arbeit mit zwei privaten Projektgruppen gelang es, die Theaterstücke „Ankunft“ und „Zeit“ zu erarbeiten und am Goethe Institut in Montreal mit Erfolg zur Aufführung zu bringen. Basierend auf den gewonnenen Erkenntnissen entstand die Unterrichtskonzeption für das Projekt Zeitumwandlung.

\subsection{Institutioneller Kontext}

Der Kurs „Literatur verstehen und inszenieren“ wird am UCC für Studierende des 3. Studienjahres angeboten. In diesem Kontext wurde das Projekt Zeitumwandlung im Herbst 2006 erarbeitet; mit einer Kursgruppe aus insgesamt sieben Teilnehmern, deren Sprachkenntnisse dem fortgeschrittenen Mittelstufenniveau (B2) des europäischen Referenzrahmens entsprachen. Neben vier Studierenden irischer Nationalität und einer Gaststudentin aus den USA nahmen ebenfalls zwei Deutsche teil, die im Rahmen des Erasmusprogramms zwei Studiensemester am UCC absolvierten.

Zur Erarbeitung des Theaterstücks standen insgesamt zwölf 100minütige Kurstreffen zuzüglich zwei 5stündigen Workshops zur Verfügung. In diesem relativ kurzen Zeitraum mussten die Studierenden für eine vom Kursleiter festgelegte Handlungsstruktur dialogische Szeneninhalte entwickeln und außerdem ausgewählte literarische Texte in das Szenario einarbeiten. Für die Aufführung stand das Granary Theatre der Universität (www.ucc.ie/granary) zur Verfügung. 


\subsection{Zur thematischen Konzeption des Stückes Zeitumwandlung}

Zeitumwandlung ist ein Theaterstück, das sich mit individueller und sozialer Wahrnehmung des Phänomens „Zeit" im Spannungsfeld von rational und emotional geprägter Weltanschauung beschäftigt. Zu Beginn des Stücks präsentieren sich kühl berechnende Protagonisten, die ihr Verständnis von den Dingen der Welt einzig und allein dem Diktat mathematisch logischer Erwägungen unterwerfen. Für sie scheinen emotional erfahrbare Lebenswelten in der Auseinandersetzung mit Fragen zur „Zeit" nicht zu existieren. Es gelingt den Akteuren zwar, bestimmte historische Begebenheiten eines Ortes (hier der Stadt Cork) mit einzelnen Jahreszahlen in Verbindung zu bringen, jedoch begreifen sie Geschichte nicht als sinnhafte Erfahrung. Der ausschließlich rational funktionierende Wissensmensch verliert die Beziehung $\mathrm{zu}$ eigenen Erinnerungen, eigenen Geschichten, zu seiner eigenen Identität. ${ }^{1}$ Um diese eigenen Geschichten wieder zu beleben verfolgt Zeitumwandlung das Ziel, für die Figuren im Stück eine lebendige Erinnerungsbrücke zwischen Vergangenheit und Gegenwart zu schaffen.

\section{Eine Fotomontage als thematischer Anstoß und als Bühnenbild}

Ein Bild ist die Dialektik im Stillstand. (W. Benjamin)

Diese digital hergestellte Bildcollage ist eng verknüpft mit der Handlung des Stückes. Durch die Einblendung während der gesamten Spieldauer wird beabsichtigt, den emotionalen Bezug der Zuschauer zur Gedankenwelt des Stücks zu etablieren. Zu Beginn der Bühnenhandlung führen die Akteure, die sich unter das Publikum gemischt haben, eine Diskussion über den Bedeutungsgehalt dieser Abbildung. ${ }^{2}$

\subsection{Darstellung der Arbeitsvorgänge im Projekt Zeitumwandlung}

Die Entwicklung eigener ,fiktiver' Figuren - Durch die Interpretation bzw. Dekonstruktion literarischer Texte und historischen Faktenmaterials eines Ortes sollen die Akteure ihrer rein rationalen Rolle entschlüpfen und eigene „emotionale“ Geschichten von selbst geschaffenen Figuren entwickeln, die anschließend erzählt und inszeniert werden. Die folgenden Punkte (1-6) erläutern diesen Prozess:

\footnotetext{
1 „Je me souviens“ - „ich erinnere mich“ prangt auf den Autokennzeichen Quebecs, jener frankokanadischen Provinz, wo ich erstmals intensiv über dramapädagogische Konzeptionen im Fremdsprachenunterricht nachdachte und mich dafür begeistern lernte. Gedächtnis transportiert historische Erinnerungen einer eigenen Geschichte, die nicht nur verstandesgemäß wahrgenommen, sondern auch gefühlsmäßig verarbeitet und gedeutet wird.

2 Siehe Anhang A.
} 


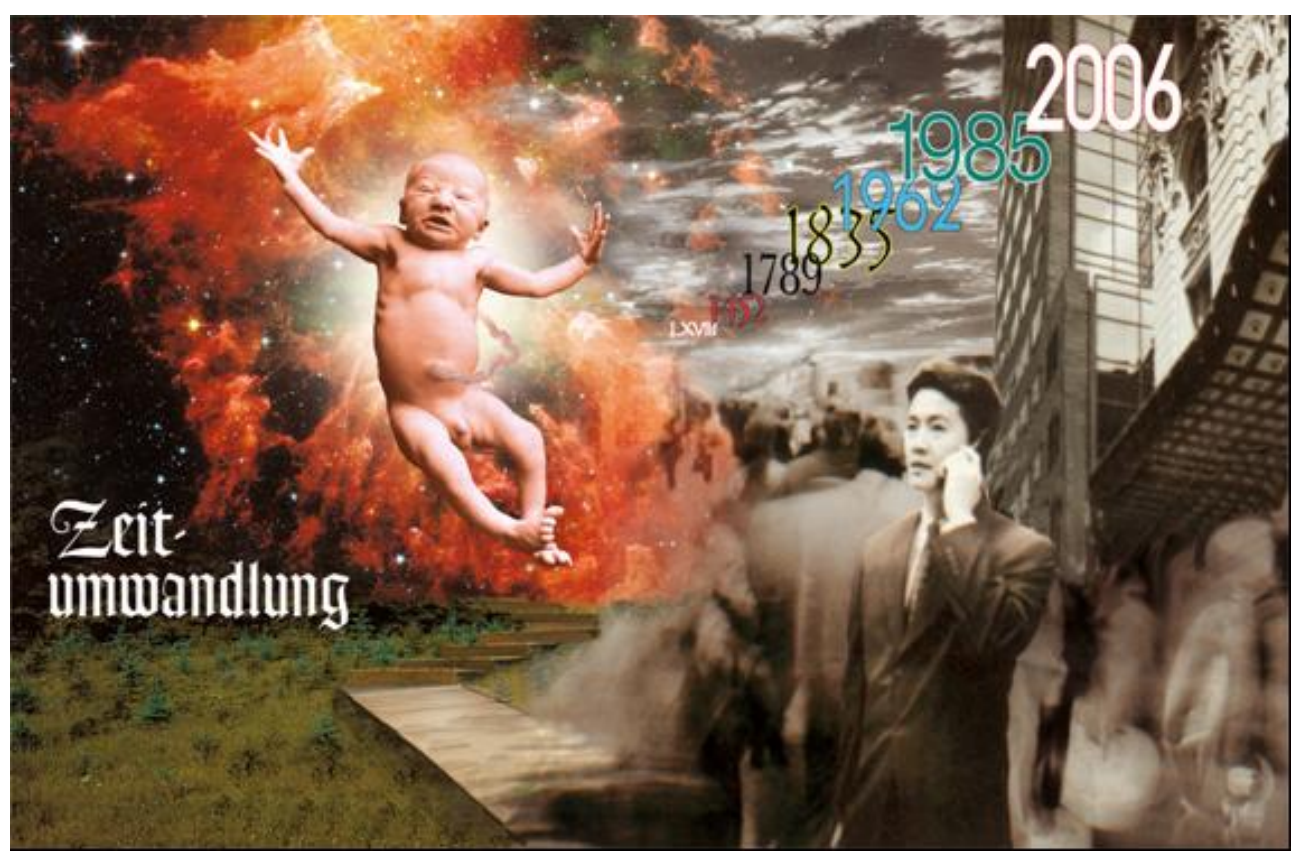

Abbildung 1: Fotomontage (@ F. Fischäss / W. Deutscher)

1. Die KT erschaffen mit Hilfe folgender Einfühlungsfragen aus Scheller (1998: 175ff) zunächst eine eigene fiktive Figur: ${ }^{3}$

- Wie heißt du?

- Wie alt bist du und wann hast du Geburtstag?

- Hast du Geschwister?

- Wo lebst du?

- Wo liegt deine Wohnung, welche Zimmer hat sie?

- Mit wem lebst du zusammen?

- Was macht ihr gemeinsam?

- Wie sah dein Alltag als Kind / als Jugendliche(r) aus?

- Was hast du an deinen Eltern gemocht, was nicht?

- Welche Ereignisse sind dir besonders in Erinnerung geblieben?

- Wovor hattest du Angst?

${ }^{3}$ Diese Auswahl wurde durch eigene, themenorientierte Fragen $\left(^{*}\right)$ ergänzt. 
- Wovon hast du geträumt?

- Wie sieht dein Alltag aus?

- Hast du einen Beruf oder eine Arbeit? Wo arbeitest du und wie lange?

- Was bedeutet dir die Arbeit?

- Interessierst du dich für Politik? Wofür besonders?

- Was machst du in deiner Freizeit?

- Was ist/wäre für dich das größte Glück / Unglück?

- Was ist deine größte Stärke / dein größter Fehler?

- Welche historischen Ereignisse empfindest du am wichtigsten?*

- Welchegeschichtlichen Personen verachtest/bewunderst du am meisten?*

Im weiteren Verlauf der Kursarbeit wird der Zugang zur Gedanken- und Wahrnehmungswelt dieses erfundenen Charakters durch verschiedene Aktionsformen intensiviert. Beim „Hot Seating“ beispielsweise sitzen die KT in der Rolle ihrer Figur auf dem heißen Stuhl und stellen sich, Bezug nehmend auf die Einfühlungsfragen, der Kursgruppe vor. Anschließend werden sie von neugierig interessierten Mitspielern „in die Zange genommen" und zu weiteren Lebensumständen vertiefend befragt. Eine interessante Sensibilisierungsreihe für sinnlich motivierte Erinnerungsarbeit findet sich ebenfalls in Boal (1989: 236f). Die Übung „Gedächtnis: Sich an gestern erinnern" lässt vor dem inneren Auge Bilder der jüngsten Vergangenheit vorbeiziehen, die körperlich nachempfunden und erspürt werden. Beispielsweise assoziieren sich mit dem Aufstehen Geräusche des Weckers oder eine menschliche Stimme, beim Duschen rinnt Wasser über die Haut oder wir begegnen beim Verlassen der Wohnung Gerüchen, Klängen, Aussagen, Stimmungen und Verhaltensweisen von Personen. Auf der Basis einer Variante dieser Übung unter dem Titel „Gedächtnis-Emotion: Ein Tag aus der Vergangenheit"notieren die KT dann stichwortartig ein entsprechendes Erinnerungsbild und ergänzen, welche positiven oder negativen Empfindungen sie mit diesem Ereignis verbinden. In Partner- und Gruppenarbeit werden die Erlebnisse geschildert und in Form eines „Hot Seating“ weiter verarbeitet.

1. Bei der Erarbeitung der verschiedenen Szenen entstehen Kommunikationssituationen, in denen die KT authentisch sprechen, indem sie für ihre Figuren Rollentexte erarbeiten, die Gefühle und Haltungen der Lernenden transportieren. 
2. Zu Beginn des Kurses erhalten die KT einen Auswahlkatalog relevanter „Routineformeln“, die die wichtigsten Teilbereiche sprachlicher Intentionen berücksichtigen. Diese Redemittel finden sich im Materialienbuch des Lehrwerks „Unterwegs“ und in „Phrases for discussion“ unter „Topics, Questions, Keywords“. Die KT sind angehalten, diese „Werkzeuge“ beim Schreiben eigener Dialog- bzw. Monologformen zu berücksichtigen.

3. Bühnenbild und Aktionsrahmen beruhen auf schlichten Gestaltungsformen und bieten Raum für individuelle Inszenierungswünsche der KT.

4. Die Szenenfolge schreibt eine Sequenz fest, die die Zuschauer über wesentliche geschichtliche Ereignisse eines Ortes informiert; sie schafft ebenfalls Freiräume für die KT, um individuell historische Fakten auswählen, bearbeiten und szenisch interpretieren zu können.

5. Die dramaturgisch festgelegte Abfolge der einzelnen Bilder erzeugt Spannung und ist thematisch so gegliedert, dass ein in sich geschlossenes und Sinn gebendes Theaterstück entsteht.

\section{Gruppenarbeit an der Fotomontage Zeitumwandlung}

Arbeitsanweisung: :

Die Zuschauer beginnen das Stück zunächst ganz alleine, denn sie werden nur das Bild Zeitumwandlung sehen, das auf die Bühne projiziert wird. Sicherlich werden diese sich jetzt Gedanken darüber machen, was das Bild ausdrücken möchte. Natürlich lasst ihr die Zuschauer nicht im Stich, denn ihr mischt euch unter das Publikum und beginnt über dieses Bild zu diskutieren. Entwickeln und schreiben Sie deshalb zu zweit oder zu dritt eine kontroverse Diskussion über das Bild Zeitumwandlung.

- Erörtern Sie dabei besonders folgende Fragen:

Welche Bedeutung könnten die dargestellten Figuren auf dem Bild haben?

Wen oder Was sollen sie repräsentieren?

Welche Rolle spielen die abgebildeten Objekte und die Jahreszahlen?

Welche Bedeutung könnte man den Farben beimessen?

- Benutzen Sie bitte für die Diskussion:

Redemittel - Übersicht

Topics, Questions, Keywords: Phrases for discussion

- Präsentieren Sie Ihre Diskussion am [Datum] vor der Gruppe 
Exkurs: Grammatik — Redeabsichten können nur durch eine adäquate Anwendung der Grammatik erfolgreich realisiert werden. Die KT sind angehalten, eigene Fehler selbstständig zu verbessern, grammatische Gesetzmäßigkeiten $\mathrm{zu}$ wiederholen bzw. zu erarbeiten. ${ }^{4}$ Im Seminar wird folgendermaßen vorgegangen:

- Eine Referenzgrammatik wird verwendet, die für alle verbindlich ist.

- Die KT reichen ihre geschriebenen Texte ein. Der Kursleiter (KL) markiert Grammatikfehler und verweist auf das entsprechende Kapitel der Referenzgrammatik.

- Ausdrucksfehler bzw. Wörter, die den Sinn verdrehen, werden vom KL korrigiert und somit visualisiert.

- DieKT erarbeiten und wiederholen grammatische Phänomene selbständig und korrigieren eigene Fehler entsprechend des Regelwerks.

- Resistente Grammatikprobleme werden im Kurs oder Einzelgesprächen diskutiert, Grammatikregeln wiederholt und Lösungsmöglichkeiten erarbeitet.

\section{Erarbeitung der Szenen von Zeitumwandlung _ -}

\section{Szene 1 - Von Kopf bis Fuß verkörpert:}

Von Kopf bis Fuß verkörpert

es haart es stirnt es nast

[es scheidet es lippt und klitorisiert]

es augt es braut und lidet

[es schwanzt und eichelt und hodet]

es mundet es lippt es zahnt und zungt

es wangt es ohrt es kinnt

es wirbelt es rückt es hintert

es hirnt und kopft

es schenkelt es kniet

es halst es schultert und brustet

es beint und fußt und ferst und zeht

es herzt

von kopf bis fuß es körpert

es armt es handet es fingert

von hirn bis herz es körpert

es baucht es nabelt es hüftet

von kopf bis fuß verkörpert

Dieser Prosatext fordert geradezu auf zur pantomimischen Interpretation und eignet sich in zweierlei Hinsicht als Einstieg ins Kursgeschehen. Die darin erwähnten Körperteile werden zum Bezugspunkt für Übungen, die eine positive Gruppendynamik fördern und die KT enthemmen, z.B. indem die KT aufgefordert werden, ungewöhnliche Begrüßungsformen vorzustellen. ${ }^{5}$

\footnotetext{
${ }^{4}$ Eine Analyse der Arbeitsergebnisse hinsichtlich der Verbesserung der Sprachrichtigkeit ist im Rahmen dieser Arbeit nicht vorgesehen. Aufgezeigt werden soll hier lediglich die Arbeitsweise, die zur Erarbeitung der verfassten Bühnentexte führt.

${ }^{5}$ Die in Klammer gesetzten Textteile werden zwar semantisch erfasst, doch steht den KT deren szenische Interpretation frei.
} 
Darüber hinaus wird eine Sensibilisierung für emotionale bzw. rationale Befindlichkeit angestrebt, was die Einfühlung in die Grundcharaktere des Bühnenstücks erleichtert.

Die Erarbeitung der szenischen Interpretation erfolgt in drei Einheiten:

Einheit 1.1 - Einfühlung - Der Kursraum ist in zwei „Welten“ unterteilt: die rationale und die emotionale. Die KT bewegen sich in beiden Räumen und nehmen dabei der Raumcharakteristik entsprechende Geh-, Sitz- und Standhaltungen ein.

Auf ein Zeichen des KL begrüßen sich die KT kühl bzw. überschwänglich. Sie erhalten Wortkarten mit den im Prosatext erwähnten Körperteilen. Sie nehmen dem jeweiligen Raum entsprechende Gehhaltungen ein, indem sie das entsprechende Körperteil im Bewegungsablauf überbetonen.

Die KT begrüßen sich mit diesen Körperteilen jeweils raumspezifisch mit frostig reservierten oder gefühlsbetont offenen Ausdrucksformen.

Einheit 1.2 - Vertiefung - Innenstirnkreis (mit der Körpervorderseite zugewandt stehen sich die KT im Kreis gegenüber): Der KL nimmt eine Karte, auf der ein Körperteil (Nomen) verzeichnet ist und versprachlicht es weiter, z.B. der Kopf - es kopft. Der KL führt anschließend mit diesem Körperteil eine übertriebene Bewegung aus und wiederholt diese mehrmals. Die Gruppe imitiert und wiederholt diesen Ablauf. Die KT erhalten weitere Wortkarten, gehen nacheinander in den Kreis und erfinden eigene Sprach- und Bewegungsformen.

Einheit 1.3 - Szenische Interpretation - Die KT lesen den Prosatext in Gruppenarbeit, diskutieren und setzen ihn szenisch um.

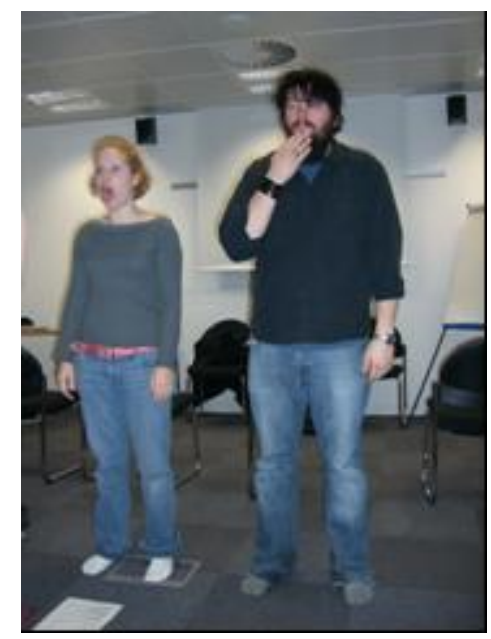

Abbildung 2: „es mundet - es lippt“

Die emotionsgeladen-sinnlichen Textzeilen sollen anschließend so verfremdet werden, dass ein gegenteiliger, rational kühler Text entsteht, der von den Akteuren ebenfalls im Bühnenstück präsentiert wird. 


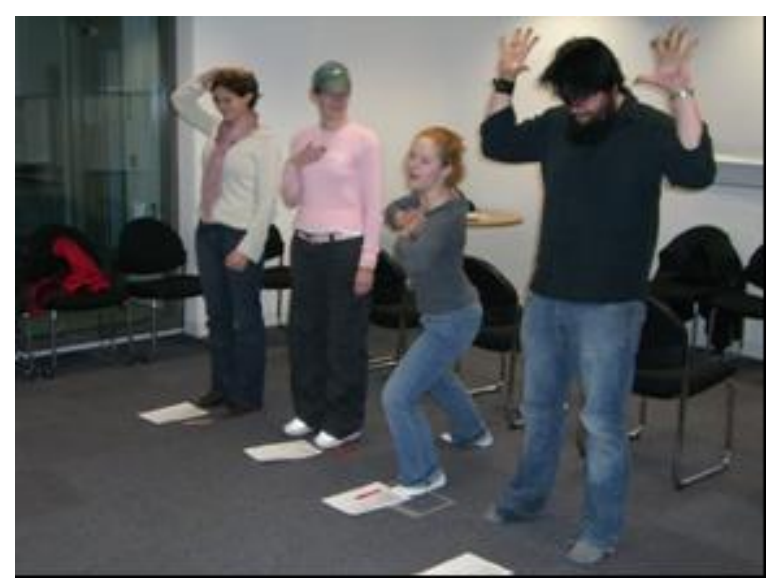

Abbildung 3: „es kopft - es herzt - es brustet - es handet, es fingert"

Szene 2 - Homo Faber: Der graue, telefonierende Herr im Bühnenbild steht in dieser Sequenz für das Charakterbild von Menschen, deren unterkühltes Denken und Handeln sich ausschließlich durch logische Berechnung erschließt. Als literarischer Repräsentant wurde der Ingenieur Walter Faber die Hauptfigur in Max Frischs Roman „Homo Faber“, gewählt. Einige seiner prägnanten Zitate ${ }^{6}$ aus dem Roman bilden den literarischen Gehalt dieser Szene und sollen mittels Passivkonstruktionen dialogisch inszeniert werden.

Einheit 2.1 - Einfühlung - Der KL gibt verschiedene Gefühlszustände vor (fröhlich, überschwänglich, betrunken, ernst, kühl, angespannt). Die KT setzen diese Emotionen in entsprechende Geh- und Sitzhaltungen um. Sie entwickeln dazu Standbilder, um diese Emotionen zu visualisieren.

Einheit 2.2 - Vertiefung - KT erhalten jeweils ein Zitat aus Homo Faber, gehen damit durch den Raum und lesen es in diesen verschiedenen Gefühlslagen. Die KT stellen fest, durch welche Gefühlszustände bzw. Körperhaltungen sich die Textinhalte adäquat ausdrücken lassen. Der KL informiert über den Roman und das Charakterbild Walter Fabers.

Einheit 2.3 - Szenische Umsetzung - KT stehen im Halbkreis und erhalten jeweils ein Zitat. Der KL hat zu den Zitaten verschiedene Passivformen vorbereitet.

Beispiel: es wird studiert, es wird gelesen, es wird gemalt

Einzelne KT reagieren darauf mit dem eigenen Zitat, das ihnen passend erscheint. Sie gehen daraufhin auf den KL zu und antworten mit diesem Zitattext kühl auf seine Ausgangsreplik. Die genannten Passivformen beziehen sich beispielsweise auf das folgende Zitat aus Frischs Roman:

\footnotetext{
${ }^{6}$ Siehe Anhang B.
} 
"Ich habe, offen gesprochen, nie daran geglaubt, dass Philologie und Kunstgeschichte sich bezahlt machen".

Wiederholungen führen in der Folge zur Intensivierung der rational gesprochenen Replik und dienen als Grundlage für die anschließende Improvisation mit weiteren Zitaten. Die KT erhalten die übrigen Faber-Zitate und entwickeln in Gruppenarbeit eine Inszenierungsform mit eigenen Dialogen.

Szene 3 - Zeitmaschinen: die Jahreszahlen im Bühnenbild: Als monoton funktionierende Apparate erschließen sich die Rationalisten den Bedeutungsgehalt der Jahreszahlen. Eine menschliche Zeitmaschine setzt sich in Bewegung und spuckt die Jahreszahlen in kühler Distanz zu den Inhalten historischer Ereignisse eines Ortes (hier der irischen Stadt Cork) aus. Dabei bleibt der perfekt arbeitende Mensch von der Emotionalität der Begebenheiten unberührt und ergötzt sich ausschließlich an seinem Sachverstand. Somit wird die Symbolik der Jahreszahlen zur Metapher eines problematischen Geschichtsverständnisses. Schließlich gerät der Motor aus dem Rhythmus und das Funktionsgefüge Mensch zerbricht.

Die Erarbeitung dieser Szene weist zwei Unterrichtseinheiten aus:

Einheit 3.1 - Historische Ereignisse - Für die Präsentation historischer Fakten über die Stadt Cork erhalten die KT eine englischsprachige Zeitleiste.

In Gruppenarbeit diskutieren die KT eine Auswahl, die präsentiert werden soll. ${ }^{7}$ Die Übersetzungsarbeit leisten die KT vorab als Hausaufgabe.

Einige Beispiele:

622: Die Stadt wurde von dem Heiligen Sankt Finbarr als ein klösterliches Zentrum gegründet.

1728: Eine Hungersnot wütet in Cork.

1849: Die Queen's Universität öffnet ihre Tore für Studenten.

1920: Oberbürgermeister MacCurtain wird am 20. März von Truppen der Krone ermordet.

2005: Cork wird Kulturhauptstadt des Jahres.

Einheit 3.2 - Entwickeln einer Zeitmaschine - Ziel dieser Einheit ist es, Bewegungsfantasie für die szenische Gestaltung einer Zeitmaschine anzuregen.

Die KT stehen in einem großen Kreis. Es wird ein „Joker“ bestimmt, der eine Bewegung beginnt und mit einem Geräusch verbindet. Andere KT imitieren Bewegung und Geräusch und beginnen auf diese Weise, sich vom „Joker“ zu entfernen. Der wiederum versucht sich anzunähern und einen anderen KT zu erhaschen. Rennen ist nicht erlaubt!! Wird ein anderer Spieler abgeschlagen, beginnt dieser eine neue Bewegung-Geräusch-Kombination und das Spiel beginnt von vorne.

\footnotetext{
7 Interessant entwickelte sich die Diskussion über die Bedeutung historischer Ereignisse, was den nicht irischen KT überraschende neue Einblicke in das Geschichtsverständnis der „Einheimischen" eröffnete.
} 
Die Rhythmus-Maschine: Ein KT bewegt sich rhythmisch auf der Stelle und stößt ein Geräusch dazu aus. Ein anderer KT tritt mit einer anderen Bewegung und Geräusch hinzu. Alle schließen sich in der Folge an, bis sich die Maschine synchron bewegt.

Die KT improvisieren verschiedene Maschinentypen, z.B. Begrüßungsmaschine, Fließband, Waschanlage, usw. Dann erarbeiten die KT unter Einbeziehung ausgewählter historischer Ereignisse eine Zeitmaschine.

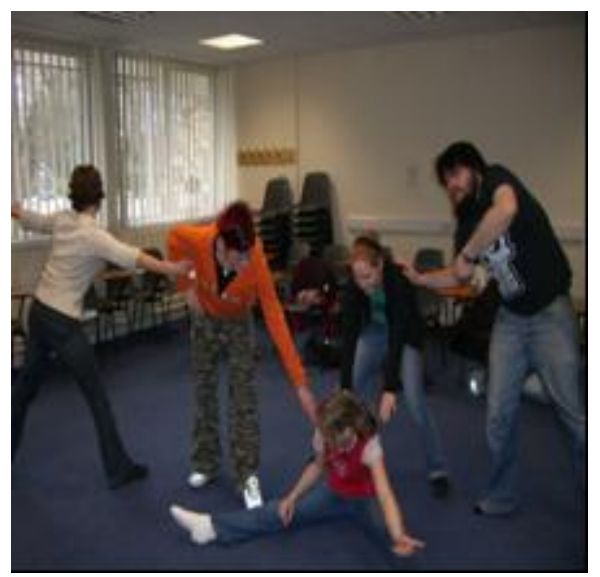

Abbildung 4: Improvisation - Die Rhythmus-Maschine

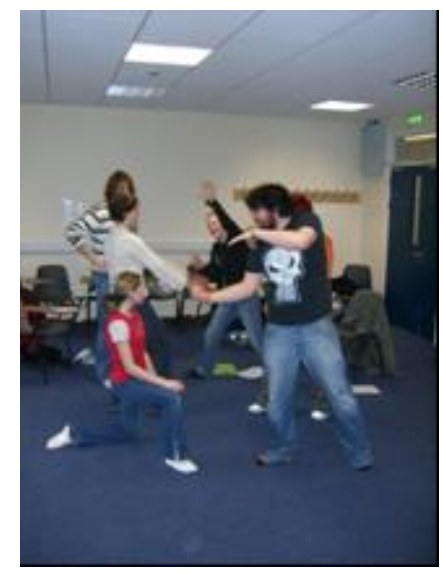

Abbildung 5: Die Zeitmaschine entsteht

Szene 4-Umgelegt : Das Baby im Bühnenbild: Vorgetragen von einer KT erhebt sich mahnend die Stimme seines noch unschuldigen, nicht manipulierten Lebens und hält den regungslos am Boden liegenden menschlichen Maschinenteilen den Spiegel vor. Als „Anklageschrift“ gegen die im Bühnenstück dargestellte fehlende Emotionalität menschlicher Existenz soll ein Textauszug aus der Kurzgeschichte „Umgelegt" ${ }^{\text {"8 }}$ von Detlev Mahnert in diese Szene eingearbeitet

\footnotetext{
8 Siehe Anhang C.
} 


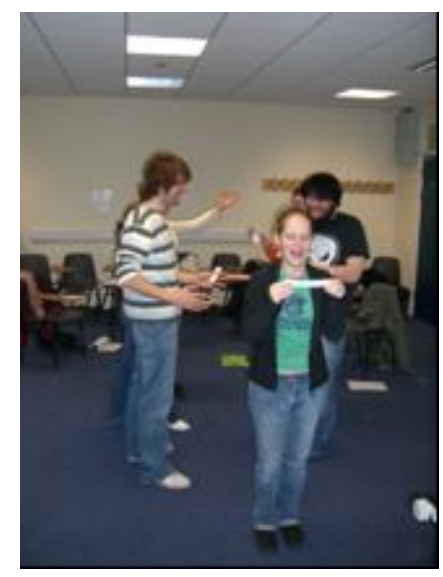

Abbildung 6: Die Zeitmaschine funktioniert

werden.

Einheit 4.1 - Textverständnis und -interpretation - Die KT erarbeiten Wortschatz und Textinhalte als Hausaufgabe. Sie stellen außerdem Vermutungen über den Sinngehalt der Textstelle „will ich hinauf in jene Höhen steigen, wo eure Gründe vor der Wirklichkeit entfliehn" an und reichen diese schriftlich ein (in der im Anhang dokumentierten szenischen Interpretation mit ** gekennzeichnet). ${ }^{9}$

Einheit 4.2 - Einfühlung in die Rolle des anklagenden Kindes - Im Raum werden Gefühlsbereiche eingerichtet (traurig, müde, zornig, enttäuscht). Die KT erhalten jeweils eine Strophe von „Umgelegt" und lesen sie in diesen Gefühlslagen. Eine vergrößerte Kopie des Erwachsenen im Bühnenbild wird in die Mitte eines Stuhlkreises gelegt. Die KT steigen auf Stühle, rezitieren die Strophen des Gedichts und richten ihre Stimme gegen diese am Boden liegende Figur. Dann tauschen die KT Gedichtstrophen aus und wiederholen die Schritte.

Szene 5 - Der Urknall: Die rote Gaswolke im Bühnenbild, wohin das Baby mit den Rationalisten „entschwebt“, symbolisiert den Urknall als Ursprung des Universums und damit auch des Lebensraumes „Erde“ und unserer irdischen Menschheits-Geschichte. Durch die explosive Expansion von Materie, Raum und Zeit soll in dieser Szene ein mögliches Bild unserer menschlichen Emotionalität entworfen werden. Wie Galaxien, die zunächst in Einklang umeinander kreisen um sich schließlich anzuziehen, können sich ebenfalls soziale Beziehungen entwickeln. Anfangs noch harmonisch, doch dann zusehends fordernder und aggressiver, kommt es schließlich zur Explosion, zum Zerwürfnis. Bindungen zerbrechen; dabei lassen sich die in der Ursachenkette ursprünglich verwobenen

\footnotetext{
9 Dieser Textausschnitt wurde vom KL ausgewählt und verändert, um einerseits eine Verbindung zur nächsten Szene „Der Urknall“ herzustellen und um andererseits die formulierten Annahmen dialogisch in die Präsentation des literarischen Textes einflechten zu können.
} 
Gedanken und Gefühlszustände oft nicht mehr detailliert nachvollziehen. Der Urknall als Allegorie menschlicher Gefühlswelten erzeugt in dieser Bühnensequenz Sprachlosigkeit, die Fragen über die eigene Identität aufwirft, und die Ausgangspunkt für emotional gesteuerte Erinnerungsarbeit ist, damit das Individuum wieder zu sich selbst finden kann.

Die Bewegungsdynamik und die Geschwindigkeit dieses Entstehungsprozesses geben den Impuls für eine tänzerische Interpretation, die sprachlich begleitet werden soll.

Imperativformen als Sprechakte sowohl sehnsüchtiger Anziehungskraft als auch aggressivem Weg- und Verstoßen begleiten die Bewegungsmuster der Akteure. Die Umsetzung dieser Szene sieht drei Einheiten vor: zwei vorbereitende als Hausaufgabe und für das Kurstreffen eine dritte zur Erarbeitung der Tanzperformance.

Einheit 5.1 - Imperativformen - Arbeitsanweisung:

Der Urknall (big bang) erwartet uns zu einem heißen Tanz. Während sich die TänzerInnen zuerst verführen und der Tanz harmonisch verläuft, werden sie sich am Ende auch sehr aggressiv begegnen, bis es dann zur Explosion kommen wird. Selbstverständlich werden die Aktionen sprachlich begleitet. Formulieren Sie deshalb mittels Imperativformen zuerst einige „liebevolle“ und „verführerische“ Aufforderungen, die dann zusehends mehr aggressive und abstoßende Formen annehmen. ${ }^{a}$

Beispiel: liebevoll - verführerisch aggressiv - abstoßend

Komm zu mir! Lass mich in Ruhe!

Massier mir den Rücken! Sei endlich ruhig!

${ }^{a}$ Textbeispiele siehe Anhang D, 1.

Einheit 5.2 - Lebensfragen -

Arbeitsanweisung: : 
Durch die Reise hinein in den Ursprung der Zeit (Urknall) haben Sie nicht nur die Entstehung der Zeit erlebt, denn Sie wurden darüber hinaus mit e i n e r Wahrheit des Lebens konfrontiert, nämlich der von Harmonie und Disharmonie. Als Kopfmensch haben Sie zum ersten Mal erfahren, welche positive und negative Kraft Emotionen haben können. Diese extreme Erfahrung hat Sie völlig verwirrt und aus Ihrer rationalen Bahn geworfen. Der Schock sitzt tief und Sie stellen sich Fragen zu Ihrem Leben. Überlegen Sie sich bitte zwei Beispiele, was „Zeit“ für Ihre Figur im Stück bedeutet. Verwenden Sie dazu bitte nur einen Artikel, ein Adjektiv und ein Nomen.

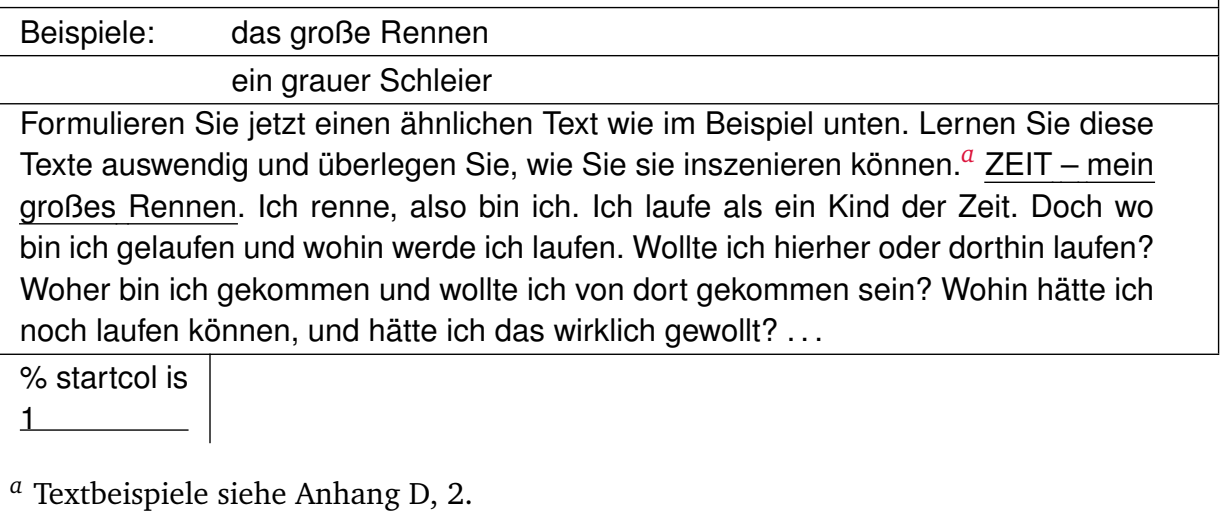

Einheit 5.3 - Sprech- und Bewegungsfantasie entwickeln - KT stehen sich in zwei Reihen in größerem Abstand gegenüber. Sie werfen sich weiche Vokale $a, e, i, o, u$ zunächst wie Softbälle zu, dann mit liebevollen Imperativformen. In einem zweiten Schritt werfen sich die KT die harten Laute $k a, k e, k i, k o, k u$ wie Stahlkugeln zu, dann mit aggressiven Imperativformen. Im nächsten Schritt nimmt je ein KT zu einem anderen Gruppenmitglied gegenüber Sichtkontakt auf. Sobald diese „optische“ Paarbildung klar ist, gehen beide aufeinander zu und begrüßen sich mit liebevollen Imperativformen, dann mit aggressiven Imperativformen

1. Bewegungsschlange (Musik: Soundtrack Kill Bill: "Battle without honor or humanity") ${ }^{10}$

Die Mitglieder der Kursgruppe stehen hintereinander in einer Reihe. Der KT an der Spitze beginnt sich zur Musik rhythmisch fortzubewegen, alle anderen folgen in exakt derselben Bewegungsart, bis der Schlangenletzte an der Gruppe vorbei an die Spitze läuft und seinerseits mit einer neuen Bewegung die Gruppe führt, usw...

\section{Handtanz (Musik: Cirque du Soleil : "Ouverture")}

Zwei Tanzpartner berühren sich mit der rechten Handfläche, wobei A führt und B folgt und umgekehrt, bis irgendwann keiner mehr führt.

\footnotetext{
10 Sämtliche Musikstücke für diese Einheit wurden vom KL aufgrund ihrer melodischen und rhythmischen Eigenschaften ausgewählt und können beliebig ausgetauscht werden.
} 
1. Wassertanz (Musik: G.E.N.E: "Life is a Melody")

Partnertanz: Einer spielt den Wassertänzer, der sich unter Wasser bewegt. Der andere spielt das Wasser, das den Tänzer umgibt.

1. Bewegungsmuster und Raumerfahrung (Musik: Chemical Brother: "My elastic eye")

KT bewegen sich in Einzel-, Partner- und Gruppenarbeit zu Bewegungsmustern, die vom KL auf folgenden Symbolkarten angezeigt werden. Dabei soll Bewegungsfantasie für Raumwege und Gruppenkonstellationen entwickelt werden, die sich für die Erarbeitung einer Tanzperformance umsetzen lassen. ${ }^{11}$

\begin{tabular}{|l|l|l|l|l|l|l|l|l|}
\hline i) & ii) & iii) & iv) & v) & vi) & vii) & viii) & ix) \\
\hline
\end{tabular}

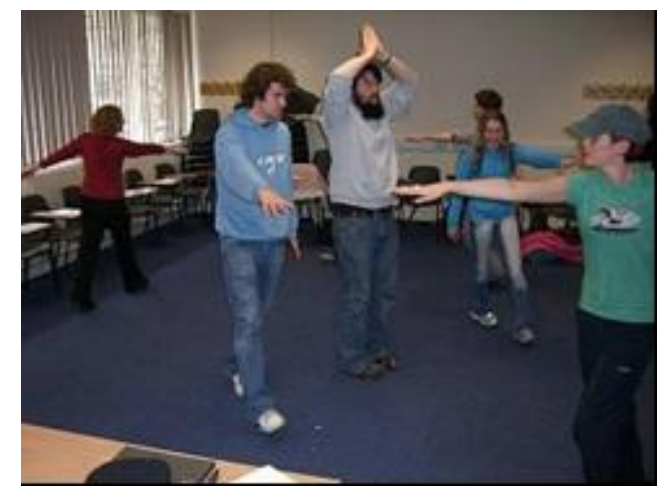

Abbildung 7: Urknall: Kreisende Galaxien im Kursraum

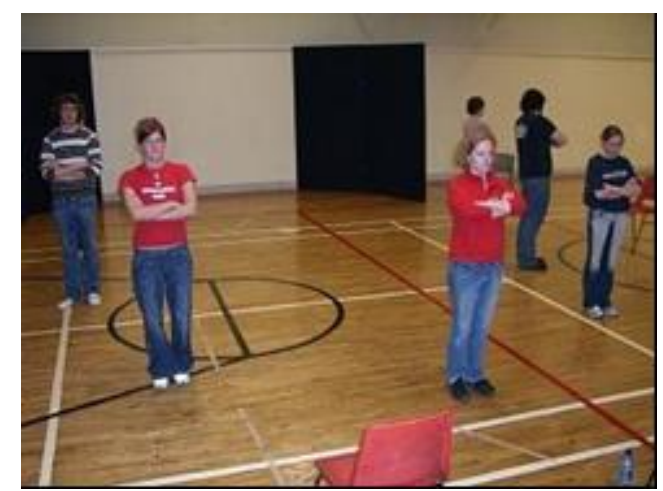

Abbildung 8: Proben der Urknall-Tanzperformance während des ersten Workshops

\footnotetext{
${ }^{11}$ Aus den Mustern viii) und ix) entwickelten die KT beispielsweise bewegende „Galaxien“, die um ein Zentrum kreisen (siehe Abb. 7).
} 
Szene 6 - Barbara Köhler: „Die Bergung“: „Die Bergung“ als literarischer Stimulus regt in dieser Szene die Gedächtniskraft der bislang rational gesteuerten Figuren an, um emotionale Erinnerungsbilder zu provozieren, die auf der Bühne erzählt werden. ${ }^{12}$ Ausgelöst durch die Interpretation dieses Textes werden Sinnes- und Körperempfindungen belebt, die Stimmungen, Erregungen, Affekte und Gemütserlebnisse verloren gegangener Episoden aus dem Leben der fiktiven Figur einfangen. Vier Unterrichtsschritte sind zur Erarbeitung dieser Sequenz vorgesehen:

Schritt 6.1 - Erinnerungen sinnlich wachrufen (Musik: Solitudes:,Exploring nature with music") - KT sitzen aufStühlen oder liegen aufdem Boden. Sie entspannen bei meditativer Musik und versuchen jedes Körperteil isoliert und bewusst zu bewegen. Der KL fordert die KT auf, die Augen zu schließen und sich an den gestrigen Tag zu erinnern. Diese Erinnerungen sollen vor allem mit körperlich-sinnlichen Empfindungen verbunden sein (z.B. ,Spaziergang': Wie verhalten sich meine Arme und Beine? Was sehen die Augen, riecht die Nase? usw.). Die KT schließen die Augen und begeben sich auf ihre eigene Zeitreise.

Schritt 6.2 - Erinnerungen erzählen und spielen - Als Hausaufgabe vorbereitet erzählen sich die KT einen wichtigen Tag aus dem Leben ihrer fiktiven Figur. Ein Partner hört zu und versucht aufgrund dieses Berichts möglichst viele Körpererfahrungen zu spielen. Es folgen Diskussion und Partnerwechsel.

Schritt 6.3 - Erfühlen des Texts „Die Bergung“ - Lesen mit den Sinnen - Nachdem der Text als Hausaufgabe vorbereitet wurde, werden zunächst offene Fragen der KT zum Textverständnis diskutiert. Die KT lesen den Text und stellen in Partnerarbeit fest, welche Textinhalte sie dabei schmecken, tasten, sehen, riechen und hören können.

Schritt 6.4 - Erinnerungen erzeugen - Der Text ist in Sinnabschnitte zerschnitten und ausgelegt. Der KL nimmt einen Textausschnitt und versprachlicht vor der Gruppe Erinnerungen aus seinem Leben, die dieser Text provoziert (Teacher in role).

Beispiel (Textausschnitt): Mein Ort heißt Kaßberg. Hier flammt kein Dornbusch, hier brennen nur winters die Müllcontainer.....

(Teacher in role): Mein Ort heißt Dudelingen, eine kleine Stadt im Süden der Bundesrepublik Deutschland. Nach einem großen Stadtrand im 17. Jahrhundert wurde sie lieblos und fantasielos wieder aufgebaut. Eine Art Hassliebe verbindet mich mit diesem Ort, denn......

KT wählen Textausschnitte aus und verfassen in Einzelarbeit Erinnerungsbilder ihrer Figuren. (Abb. 9 und 10). Dann stellen die KT stellen einzelne Bilder in einem Kurzvortrag vor und verfassen weitere Erinnerungsbilder als

\footnotetext{
${ }^{12}$ Gesamttext mit Erinnerungsbildern der Figuren siehe Anhang E.
} 
Hausaufgabe. Eine Diskussion sowie die Umsetzung von Inszenierungsideen für diese Bilder erfolgt beim nächsten Kurstreffen.

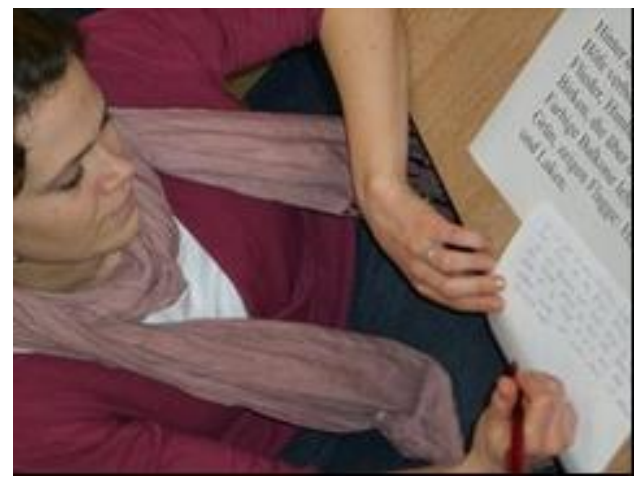

Abbildung 9: Die Bergung: Erinnerungsbilder entstehen

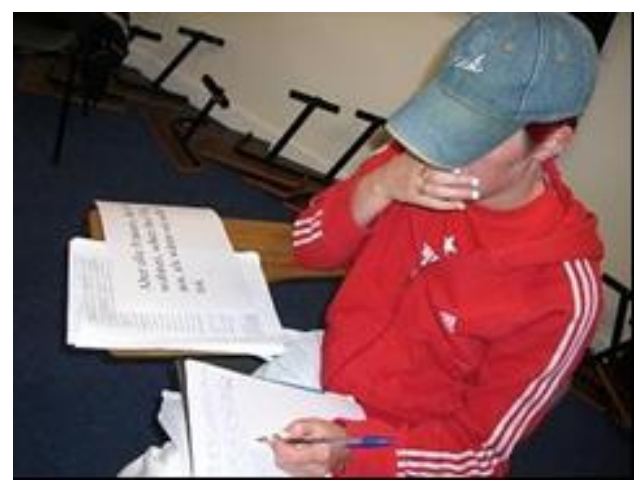

Abbildung 10: Die Bergung: je me souviens - ich erinnere mich

Szene 7 - Historische Zeitumwandlung: Die Transformation des Verstandesmenschen zu einem emotional geprägten Individuum findet ihren Fortgang in historischen Geschichten, die weitere Erinnerungen ins Bewusstsein der Figuren zurücktransportieren. Was die Akteure zuvor noch an der kühl distanzierten Betrachtungsweise geschichtlicher Ereignisse zerbrechen ließ (Szene 3 - Zeitmaschinen), soll nun durch eine emotional geprägte Interpretationsform historischen Faktenmaterials abgelöst werden.

Nachdem die KT über einen literarischen Text an das Verfassen von Erinnerungsbildern herangeführt wurden, kann ein analoges Interpretationsverfahren nun auf historische Begebenheiten, die das Leben der irischen Stadt Cork prägten, übertragen werden. Zunächst wählt jeder KT für seine Figur individuell einige dieser Geschichten aus, übersetzt den englischen Originaltext aus „CORK 365 - a day-by-day miscellany of Cork History“ und stellt diesen vor der Gruppe vor. Anschließend entwickeln die KT in Gruppenarbeit eine Szene, in die ausgewählte historische Inhalte eingearbeitet werden sollen.

Aus der folgenden Arbeitsanweisung für eine Hausaufgabe resultiert das 
folgende Szenario „Cork Radio“13, das schließlich zur Schlussszene des Stücks überleitet.

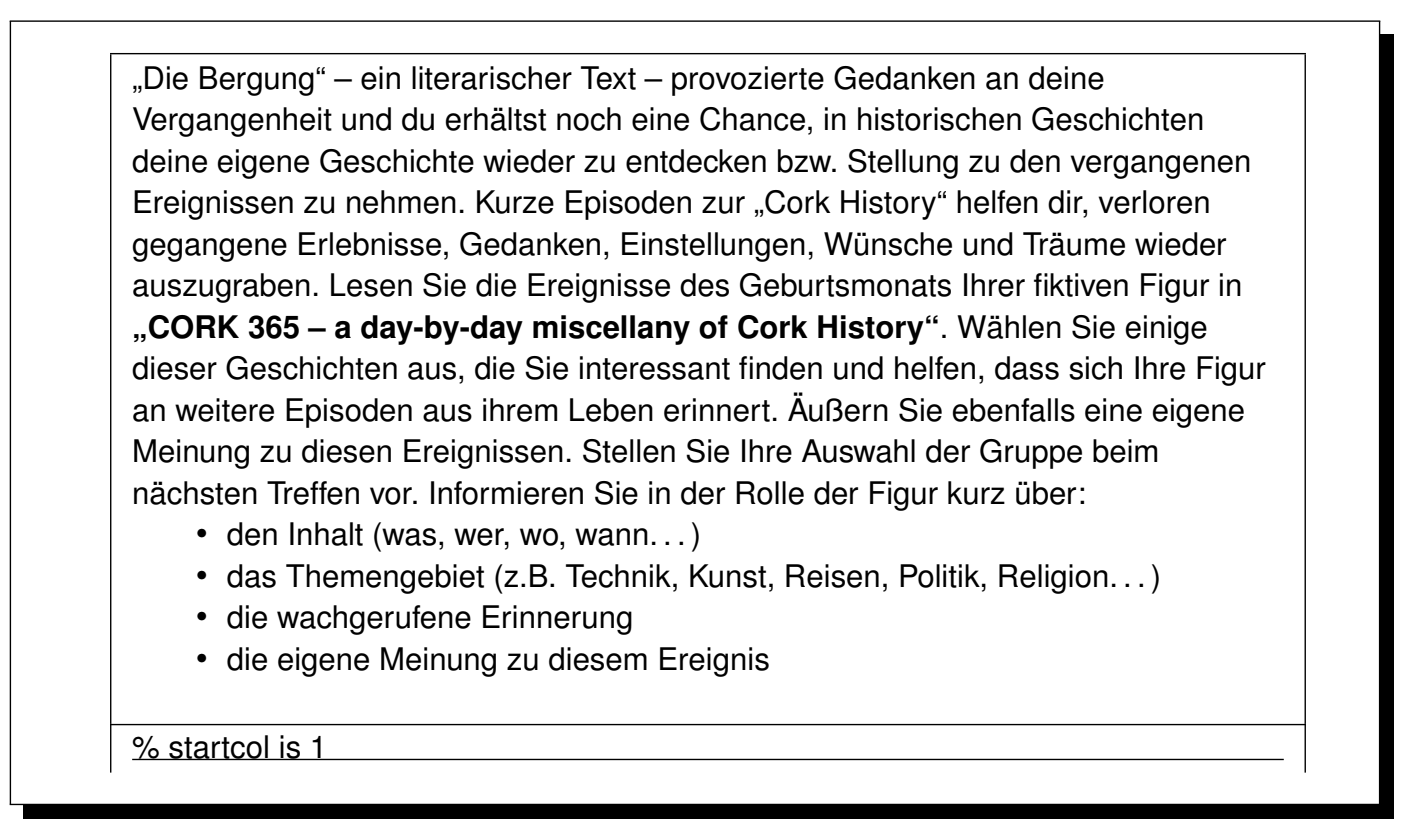

Schlussszene - „Von Kopf bis Fuß verkörpert“ : Die szenischen Konfrontationen mit dem Phänomen „Zeit"sowie historisch - literarischen Inhalten haben bei den anfangs kühl und nüchtern (be)urteilenden Akteuren erste Sinneswandlungen bewirkt und zu gefühlsbetonteren Interpretationen individueller Wahrnehmungswelten geführt. Der Kreis im Theaterstück Zeitumwandlung schließt sich nun, denn aus den von ausschließlich kopfgesteuerten Rationalisten entwickeln sich Menschen, die ihre Lebenswelt zusehends emotional, nämlich von „Kopf bis Fuß verkörpert" erleben. Am Ende des Stücks steht deshalb die Präsentation dieses literarischen Textes, zu dem eine Überleitung geschaffen werden sollte, wo sich die Akteure nochmals direkt an das Publikum wenden. Sie liest sich wie folgt: ${ }^{14}$

Laura: Bemerken Sie, wie sehr Sie sich selbst und ihre Umgebung verändert haben!

Estella: Landen Sie dort, wo Menschlichkeit und moralisches Handeln und Denken in einer kapitalistischen Gesellschaft nicht untergehen! Harm: Umarmen Sie die gesamte Welt!

\footnotetext{
13 Siehe Anhang F.

14 Aufgrund von Zeitmangel wurden hier kurze imperativische Äußerungen geformt. Abgeleitet aus studentischen „Quellentexten“ stammen sie aus einer Hausaufgabe, in der Vermutungen über den Textauszug „Umgelegt“ von D. Mahnert angestellt wurden. Sie möchten den Zuschauern ebenfalls Mut machen, den Lebensalltag nicht ausschließlich „kopfgesteuert“ zu gestalten, sondern auch emotionale Befindlichkeiten zuzulassen und ihnen Ausdruck zu verleihen. Eine weiterführende Auseinandersetzung mit den Inhalten dieser Äußerungen kann im Rahmen der Bühnenhandlung nicht stattfinden, denn es sollen lediglich Denkanstöße gegeben werden.
} 
Irene: Wenn ich nicht irre, sind Schlafen und Träume [... ] nicht rational, sondern ganz emotional. Also schlafen und träumen Sie einfach!

Sabine: Verwandeln Sie die Erde in eine emotionalere Welt!

Jack: Zeigen Sie, wer Sie wirklich sind!

Carl: Zeigen Sie ihre wahren Gefühle!!

von kopf bis fuß es körpert

von hirn bis herz es körpert

von kopf bis fuß verkörpert

ENDE der Zeitumwandlung

\subsection{Workshops}

Der Fokus der beiden 5stündigen Workshops (WS), die zum Ende des Kurses durchgeführt wurden, ist die konkrete Probenarbeit; also die Arbeit mit der Figur und das Verknüpfen der bis zu diesem Zeitpunkt erarbeiteten Szenen stehen im Mittelpunkt (WS 1: Szenen 1-4 / WS 2: Szenen 5-7 und Entwickeln der Schlussszene). Diese Erarbeitungsphasen mit den bereits verfassten Rollentexten soll den KT ein Gefühl für tatsächlich erworbene Textsicherheit vermitteln und gegebenenfalls zu gründlicherem Auswendiglernen dieser Texte motivieren.

Außerdem wird mit den Dimensionen und der Gestaltung des Aufführungsraums vertraut gemacht, um Positionen und Aktionswege der Akteure auf der Bühne zu etablieren. Darüber hinaus müssen sämtliche benötigten Requisiten zusammengestellt und vor allem im Verlauf von WS 2 Beleuchtungs- sowie akustische Einstellungen fixiert werden.

\section{Reflexion und Ausblick}

Das Projekt Zeitumwandlung fördert das Literaturverständnis der Studierenden und eröffnet neben dem persönlichen Zugang zu dem existenziellen Phänomen „Zeit" auch Freiräume zur Realisierung authentischer Sprechabsichten. Die Auseinandersetzung mit Szeneninhalten schafft Bedingungen für selbstkritisches und konstruktives Experimentieren in der Fremdsprache und transformiert deren lexikalische Bestandteile in erlebbare und Sinn gebende Kommunikationseinheiten.

Die Montage von Einzelbildern in eine vorgegebene Handlungsstruktur erfüllte die Erwartungen des KL, so dass sich ein aussagekräftiges und Sinn vermittelndes Theaterstück komponieren ließ. Trotz der knapp bemessenen Unterrichtszeit können die erzielten Arbeitsergebnisse durchaus positiv bewertet werden: dazu zählen vor allem die umfangreiche Ausarbeitung der Szeneninhalte sowie die beinahe einstündige Präsentation des Theaterstücks. Besonders motivierend wirkte sich die geforderte Aufführung aus, denn alle Akteure wollten sich möglichst textsicher präsentieren, was in intensive Bemühungen um eine korrekte Aussprache mündete. Besonders hervorzuheben ist die Gruppendynamik, die sich in der gemeinsamen Entwicklungsarbeit von 
Figuren, Inhalten und Inszenierungsformen äußerst produktiv entwickelte und die Selbstdisziplin der KT stärkte.

Allerdings sollte für zukünftige Realisierungen eine Erweiterung des Stundenumfangs unbedingt erwogen werden, damit die Entscheidungskompetenz der KT bei der Auswahl literarisch-musikalischer Inhalte gestärkt und deren Einsatz im Stück von den Studierenden selbst getroffen werden kann.

Diese erste erfolgreiche Zeitumwandlung mündet für den Autor in weiterreichende ,Fantasien für dramapädagogische Freiheit':

Es könnte eine besondere Kursstruktur entwickelt werden, die Spracherwerb und Literaturunterricht vernetzt und sich durchaus auch über zwei oder sogar mehrere Semester erstrecken könnte.

Vorstellbar wäre ebenfalls eine Initiative für interdisziplinäre Zusammenarbeit der Fachbereiche Sprach- und Theaterwissenschaften sowie Geschichte, denn durch kooperierende Fachkompetenz ließe sich Entwicklung und Darstellung der Stücke optimieren.

Zur Förderung von Öffentlichkeitsarbeit könnten der lokalen Bevölkerung durch Zeitumwandlung vertiefend Fakten ihrer eigenen historischen Wurzeln präsentiert werden. So entstünde für dramapädagogische Unterrichtsarbeit eine mögliche Plattform zur Außendarstellung und somit ein Forum für öffentliche Wahrnehmung ganzheitlicher Unterrichtspraxis im Fremdsprachenunterricht.

\section{Bibliographie:}

Beecher, Seán (1992): Cork 365: A Day-by-Day Miscellany of Cork History. Cork: The Collins Press

Boal, Augusto (1989): Theater der Unterdrückten: Übungen und Spiele für Schauspieler und Nicht-Schauspieler. Frankfurt/M.: Suhrkamp

Boal, Augusto (1997) : Jeux pour acteurs et non-acteurs: Pratique du théâtre de l'opprimé. Paris: La Découverte

Dreyer, Hilke / Schmitt Richard (2002): Lehr- und Übungsbuch der deutschen Grammatik: A Practice Grammar of German. New edition, 1. Ausgabe. Ismaning: Verlag für Deutsch

Even, Susanne (2003): Drama Grammatik: Dramapädagogische Ansätze für den Grammatikunterricht Deutsch als Fremdsprache. München: iudicium

Fischäss, Frank (2000): Sprechakte - Sprachspiel - Szenisches Spiel: Zur pragmatischen Begründung des Fremdsprachenunterrichts. Montreal: McGill University. <http: //www.collectionscanada.ca/obj/s4/f2/dsk2/ftp03/M064149.pdf>

Frisch, Max (1997): Homo Faber: Ein Bericht. Frankfurt/M.: Suhrkamp Hachenburger, Petra / Jackson, Paul (2000): Topics, Questions, Keywords: A Handbook for Students of German. London and New York: Routledge

Köhler, Barbara: Die Bergung. 10. Juni $2007<$ http://www.tuchemnitz.de/phil/germanistik/chemnitzer_autoren/autor/koehler.html> 
Mahnert, Detlev: Umgelegt. 10. Juni 2007

<http://www.e-stories.de/view-kurzgeschichten.phtml?4837>

Mettenberger, Wolfgang (1993): Tatort Theater: Ein praktischer Leitfaden für die Schul- und Amateurbühne. Offenbach: Burckhardthaus-Laetare

Materialienbuch (1998). In Lehrwerk „Unterwegs“. München, Berlin. Langenscheidt

Neelands, Jonothan (1990): Structuring Drama Work: A Handbook of Available Forms in Theatre and Drama. New York: Cambridge

Polsky, Milton E. (1998): Let's Improvise: Becoming Creative, Expressive and Spontaneous through Drama. New York, London: Applause Theatre Books

Pura, Talia (2002): Stages: Creative Ideas for Teaching Drama. Winnipeg: J.

Gordon Shillingford

Scheller, Ingo (1998): Szenisches Spiel: Handbuch für die pädagogische Praxis. Berlin: Cornelsen Scriptor

Schewe, Manfred (1993): Fremdsprache inszenieren: Zur Fundierung einer dramapädagogischen Lehr- und Lernpraxis. Oldenburg: Zentrum für pädagogische Berufspraxis, Carl von Ossietzky Universität

Sykora-Bitter, Claudia: Von Kopf bis Fuß verkörpert. 10. Juni 2007 <http://www.podiumliteratur.at/texte/107syk.htm>

Tselikas, Elektra I. (1999): Dramapädagogik im Sprachunterricht. Zürich: Orell Füssli

Vlcek, Radim (1997): Workshop Improvisationstheater: Übungs- und Spielesammlung für Theaterarbeit, Ausdrucksfindung und Gruppendynamik. München: J. Pfeiffer 


\section{A Anhang A}

\section{A.1 Bühnenbild „Ein Bild ist die Dialektik im Stillstand“"}

Interpretation zweier fremdsprachlicher KT

Sabine: Natürlich machen wir uns Gedanken über das Bild. Wenn ich mir dieses Bild so ansehe, dann bin ich überrascht. Welche Bedeutung haben die dargestellten Figuren auf dem Bild?

Laura: Meiner Meinung nach gibt es hier zwei verschiedene Welten. In der farbigen Welt geht es um die Evolution der Zeit und die andere zeigt die heutige Zeit. Was denkst du darüber?

Sabine: Selbstverständlich könnte es so sein wie du denkst, aber ich glaube, dass das Baby ein Symbol für das Heute sein könnte. Es scheint mir, dass wir heutzutage farbige Bilder benutzen um über das Heute zu erzählen. Das gilt auch im Vergleich zur Situation vor z.B. 100 Jahren, wo alle Bilder nur schwarzweiß und farblos waren.

Laura: Aber nehmen wir an, dass das Baby ein religiöses Symbol ist. Ich denke, dass es hier einen Kometen gab. Er ist explodiert und ein Kind wurde geboren. Der Hintergrund zeigt eine wilde natürliche Umgebung. Es ist Nacht und man kann die Sterne sehen. Auf einmal kommt es zu einer Explosion.

Sabine: Es wäre naiv zu glauben, dass es ein religiöses Symbol ist.

Laura: Warum nicht? Das ist nur meine Meinung!

Sabine: Es ist doch fraglich, ob das Baby tatsächlich etwas Religiöses darstellt oder mehr mit dem Mann auf der rechten Seite zu tun hat. Vielleicht sind sie sogar Verwandte?

Laura: Vielleicht repräsentieren sie, wie die Zeit sich verändert hat. Ohne Zweifel gibt es einen großen Unterschied zwischen der heutigen Zeit und der vergangenen Zeit. Zeitumwandlung steht im Bild, deshalb denke ich, dass es darum geht. Was glaubst du?

Sabine: Ich stimme zu und denke auch, dass die Farben auch eine große Rolle spielen.

Laura: Ja, ich auch. Zum Beispiel repräsentieren die Farben die emotionale Welt, wo man sich ausdrücken kann.

Sabine: ......und Schwarzweiß repräsentiert die rationale Welt, oder? Laura: Ja, das könnte sein, aber was sollen die Jahreszahlen bedeuten? Sabine: Sie sind die Jahreszahlen, die den Anfang der Zeit bis in die Gegenwart zeigen; deshalb trägt dieses Bild den Titel Zeitumwandlung'. Laura: Genau, ich denke dasselbe, und dass sich die Welt langsam verändert hat. Früher war das Leben ganz einfach und angenehm im Vergleich zum Heute, wo es sehr stressig ist. 


\section{B Anhang B}

\section{B.1 Max Frisch, Homo Faber - sämtliche Zitate}

- Ich glaube nicht an Fügung und Schicksal, [... ich bin] gewohnt mit den Formeln der Wahrscheinlichkeit zu rechnen.

- Warum soll ich erleben, was gar nicht ist?

... man isst im Gehen und verliert keine Zeit.

... wiejedeFrau eigentlich nurwissen möchte, wasich fühle, beziehungsweise denke, wenn ich schon nichts fühle. .

- Ich mache mir nichts aus Folklore.

... Man landet bei mir nicht mit Malerei und Theater.

... Radio [stelle ich natürlich] sofort ab, weil unpassend, weil Tanzmusik.

... ich war noch nie in der Opéra gewesen.

- Ich habe, offen gesprochen, nie daran geglaubt, dass Philologie und Kunstgeschichte sich bezahlt machen.

.. ich rede nicht von persönlichen Dingen.

- Überhaupt der ganze Mensch! - als Konstruktion möglich, aber das Material ist verfehlt: Fleisch ist kein Material, sondern ein Fluch.

- Ich wurde sentimental, was sonst nicht meine Art ist,...

- Ich bin gewohnt, allein zu reisen. Ich lebe, wie jeder wirkliche [Mensch], in meiner Arbeit. Im Gegenteil, ich will es nicht anders und schätze mich glücklich, allein zu wohnen, meines Erachtens der einzigmögliche Zustand für [Menschen]....

- Ich bin nicht zynisch. Ich bin nur, was Frauen nicht vertragen, durchaus sachlich.

- Meine erste Erfahrung mit einer Frau, die allererste, habe ich eigentlich vergessen, das heißt, ich erinnere mich überhaupt nicht daran, wenn ich nicht will.

- Ich kann nicht die ganze Zeit Gefühle haben. Alleinsein ist der einzigmögliche Zustand für mich [...]

- Ich kann es nicht ausstehen, wenn man mir sagt, was ich zu empfinden habe. 
Habe ich von der Zukunft etwas zu erwarten, was ich nicht schon kenne?

- Es [geht] mir, versteht sich, um genaue Daten.

Sie erwarten ein Kind. Sind Sie sicher? Immerhin eine sachliche und vernünftige Frage. Sind Sie beim Arzt gewesen. Ebenfalls eine sachliche und erlaubte Frage.

- Ich habe mich schon oft gefragt, was die Leute eigentlich meinen, wenn sie von Erlebnis reden. Ich bin [... ]gewohnt, die Dinge zu sehen, wie sie sind. Ich sehe alles, wovon sie reden, sehr genau; ich bin ja nicht blind.

Es gilt , allerlei kindische Vorstellungen vom Roboter $\mathrm{zu}$ widerlegen, das menschliche Ressentiment gegen die Maschine, das mich ärgert, weil es borniert ist, ihr abgedroschenes Argument: der Mensch sei keine Maschine.

Handlungen kann eine Maschine ebenso gut erledigen kann wie ein Mensch, wenn nicht sogar besser [...], weil sie, die Maschine, nichts vergessen kann, weil sie alle eintreffenden Informationen, mehr als ein menschliches Hirn erfassen kann....

... ganz abgesehen davon, dass es immer Freude macht, Maschinen in Betrieb zu sehen.

Max Frisch: „Homo Faber“ - Textbeispiele

- Es wird gemalt, es wird Theater gespielt.

- "[...] Man landet bei mir nicht mit Malerei und Theater."

- Es werden Dinge getan, die nur ein Mensch machen kann.

- "[Handlungen kann] eine Maschine ebenso gut erledigen [...] wie ein Mensch, wenn nicht sogar besser [...], weil sie, die Maschine, nichts vergessen kann, weil sie alle eintreffenden Informationen, mehr als ein menschliches Hirn erfassen kann [...] ganz abgesehen davon, dass es immer Freude macht, Maschinen in Betrieb zu sehen.“ 


\section{Anhang C}

\section{C.1 Detlev Mahnert, Umgelegt - Szenische Realisierung}

Untenstehend die szenische Interpretation, wie sie von den Studierenden entwickelt wurde. Die Inszenierungsanweisung zeigt die Namen der fiktiven Figuren.

(steht vor dem Baby) Seht her, seht her - ein Brief meines jüngsten neugeborenen Enkels für euch!! (sie steht auf einen Stuhl und liest anklagend den Brief bis „will ich hinauf in jene Höhen* steigen, wo eure Gründe vor der Wirklichkeit entfliehen")

Ihr, die ihr meint, stets alles zu verstehen und euch zu Richtern wohl berufen glaubt, habt mich verurteilt, und so ist mir Recht geschehen, und ich bin nicht mehr da, der euch die Ruhe raubt. Ihr wisst mich sicher hinter Mauern, hinter Gittern, ihr tötet mich und fühlt euch ganz im Recht. Ihr wisst, nun müsst ihr nicht mehr vor mir zittern - ihr seid so gut - und findet mich so schlecht...Ich will die Türen schließen hinter meinem Schweigen. Mit meinen Schattenbildern, meinen Fantasien will ich [hinauf in jene Höhen]* steigen, wo eure Gründe vor der Wirklichkeit entfliehn.

Jack: Welche Gründe entfliehen vor der Wirklichkeit?**

Carl: In welche Höhen möchte es steigen?***

Sabine: Unser Baby will wohl aufsteigen in die Höhen der Unabhängigkeit, um sich abzunabeln von seiner Mutter.***

Laura: Das Baby will wahrscheinlich eine Menge Geld verdienen, um ein leichtes und angenehmes Leben zu haben.**

Estella: Ich könnte mir vorstellen, dass das Baby sich aufmacht, um in die Sphären des Karrierismus einzusteigen.**

Harm: Wenn ich nicht irre, will das Baby in die Genforschung einsteigen, um Menschen mit Technik zu vernetzen. Dadurch würden sie noch schneller und effektiver.**

Irene: (liest den Text bis zum Schluss sehr emotional)

Was wisst ihr denn von meinen Wünschen, meinen Träumen, von meinen Ängsten, meinem neuen Mut, von meiner Furcht, das Leben zu versäumen, von meiner Zärtlichkeit, von meiner Wut? Ihr habt es nie verstanden, mich zu lieben, ihr habt auch nie verstanden, wer ich bin. Ihr habt geurteilt, habt mich aus der Welt vertrieben, und meine Todesangst verwirrt euch nicht den Sinn. Ich wollte lieben, doch ihr habt mich übersehen, und auch mein Schreien hat euch nicht gestört... Ihr, die ihr meint, stets alles zu verstehen, habt nichts verstanden, nichts gesehen, nichts gehört.

Irene: Ich könnte mir vorstellen, dass es ganz gern „über dem Regenbogen“ fliegen will. „Jene Höhen“ könnten die emotionalen Höhen des Herzens 
sein. Wollt ihr die wahren Gefühle dieser Welt kennen lernen, dann steigt hinauf in den Ursprung der Zeit. Erlebt den Urknall, empfindet die Harmonie und erlebt die Disharmonie des Lebens.

* im Originaltext: ,hinab in jene Tiefen'15

15 Die Abwandlung in „Hinauf in jene Höhen“ soll das im Bühnenbild dargestellte Hinaufschweben des Babys in die Gaswolke sprachlich illustrieren und die Vorstellungskraft von KT bzw. Zuschauern anregen, um einfacher einen Bezug zur folgenden Szene 5 „Der Urknall“ herstellen zu können. 


\section{Anhang D}

\section{D.1 Der Urknall}

1. Textbeispiele: Einheit 1 - Imperativformen

$\begin{array}{ll}\text { Nimm meine Hand! } & \text { Fass' mich nicht an! } \\ \text { Vergiss mich nie! } & \text { Halt die Klappe! } \\ \text { Spiel mir ein Lied! } & \text { Verpiss dich! } \\ \text { Erobere mich! } & \text { Verlass mich! } \\ \text { Hilf mir bitte! Hab mich lieb! } & \text { Mach, dass du wegkommst! } \\ \text { Schau mir in die Augen, Kleines! } & \text { Lass mich allein! } \\ \text { Hab Geduld! } & \text { Such dir jemand anderen! }\end{array}$

2. Textbeispiele: Einheit 2 - Lebensfragen (fremdsprachliche KT)

Zeit - die unaufhörliche Veränderung.

Jung oder alt, man kann Veränderungen bemerken. Die wechselnde Welt, wechselnde Leute und die wechselnde Zeit. Wann haben diese Veränderungen begonnen und wann werden sie enden? Wollte ich mich auch ändern? Vielleicht habe ich mich schon verändert - meine Gedanken, meine Erinnerungen, meine Träume. Wenn ich das nur wüsste, könnte ich etwas, könnte ich mich verändern! Oder vielleicht ist es schon zu spät?

Zeit - mein schnell fließender Fluss.

Der Fluss wurde geboren, das wissen wir. Irgendwo hoch in den Bergen oder vielleicht oben im Himmel in den Wolken. Wir alle sind Teile des großen Flusses, aber wir sind nur kleine Tröpfchen, deswegen können wir seine Richtung nicht ändern. Er nimmt uns mit auf seiner Reise, obwohl wir weder das Reiseziel noch die Richtung kennen. Wir fließen schnell zusammen und wissen nicht, was um die nächste Kurve liegt. Schließlich wird diese Reise enden und der Fluss wird ins Meer fließen, aber wird das das Ende sein oder wird es noch einen Anfang geben? 


\section{E Anhang E}

\section{E.1 Barbara Köhler, Die Bergung mit Erinnerungsbildern der Figuren}

Mein Ort heißt Kaßberg. Hier flammt kein Dornbusch, hier brennen nur winters die Müllcontainer.

Jack: Mein OrtheißtBoston. KeineNatur existierthier.Es gibtnur Abfallbehälter und Verunreinigung zu sehen und zu riechen.Den Sternen nicht näher, doch der Himmel drückt tiefer manchmal. [...] Manchmal sieht man die Sterne, aber meistens gibt es zu viel Smog, um sie zu sehen. Ich will immer die Sterne sehen. Die Sterne erinnern mich daran, als ich am Stadtrand wohnte.

Es gelten die Regeln deutscher Grammatik: Würde ist ein Konjunktiv.

Sabine: Ich habe die Regeln deutscher Grammatik in der Schule schon gelernt. Konjunktiv - ja, den kenne ich sicher. Es geht um Möglichkeiten und Irrealität.Aber Geografie ist noch immer die letzte Zuflucht der Mythen, und wo Gott sich ausschweigt, sprechen die Orte für sich. Auf Namen ist längst kein Verlass mehr; obwohl es berg heißt, ist kein Tal abzusehen, kein Blick übers Land, nichts zu Füßen ... Verstellte Aussichten: das Haus gegenüber, uneinsichtige Fenster, verborgene Leben, bröckelnder Putz.

Blaues Flackern: TV.

Harm: Ich erinnere mich noch genau. Als ich noch ein kleiner Junge war, kauften meine Eltern einen Fernseher. Ich konnte ewig dasitzen und darüber nachdenken, wie denn die kleinen Menschen in den Kasten kommen. Und eines Tages ging ich hin zum Fernsehschrank und steckte meinen Kopf hinein. Guckte an der Rückseite des Fernsehers. Wenn ich daran denke, kann ich immer noch den Geruch von aufsteigendem Staub in der Nase fühlen. Einmal im Monat schreit dort nachts eine Frau, als würde sie umgebracht. Aber alle Frauen, die da wohnen, sehen bei Tag aus, als wären sie schon tot.

Jack: Während der Nacht kann man die Schreie anderer Leute hören. Größtenteils sind das die Schreie der Frauen. Aber diese Frauen sehen aus, als wären sie schon tot. Keine Gefühle, keine Träume.

Karl: Als ich jung war, habe ich in einem armen Gebiet in London gewohnt. Ich besuche dort manchmal meine Freunde und ich sehe immer eine Menge von ledigen Müttern, die aussehen, als ob sie nicht mehr am Leben wären. Viele Kinder, keine Hilfe und keine Chancen. Jedes Mal, wenn ich diese armen Frauen sehe, erinnert mich das an meine eigene Mutter. Deshalb besuche ich sie nicht so oft wie vorher.

Aufgebläht wie Ophelia, Treibgut inmitten gestrandeter Träume.

Am Meer lebte ich gern, an den Grenzen des Landes, aller Sicherheit. 
Harm: Nach dem Tod meiner Familie wusste ich nicht mehr, was ich tun sollte. Ich stand tagelang am Meer und starrte auf die See hinaus. Ich dachte nach. Versuchte zu entscheiden, was ich tun sollte. Dann kam die Entscheidung. Ich wollte weg, weg aus dem Land, aus meiner gewohnten Umgebung. Auf einmal war das Meer eine Möglichkeit, der Weg zu entfliehen, zu entkommen.

Estella: Ich habe den größten Teil meiner Kindheit am Meer verbracht. Mein Vater war Fischer, wir lebten also am und von dem Meer. Ich konnte stundenlang am Strand entlang laufen, auf der Suche nach Muscheln und anderen Schätzen des Meeres. Kaum zu Hause angekommen, habe ich meine neuen Schätze gründlich gesäubert und sie stolz meinen kleineren Geschwistern präsentiert.

Die Zeit ist vergangen, wohin nur.

Sabine: Ich kann mich gerade an meine Kindheit erinnern. Vielleicht, weil ich so jung war, habe ich nicht gedacht, dass es wichtig wäre, sich an alles zu erinnern. Ich weiß nicht. Alles, was ich weiß, ist, dass ich glücklich war und dass ich viele Freunde hatte, mit denen ich spielen konnte.

Warten und Gegenwarten nehmen anderswo Platz, reichen nicht bis hierher. Im Lichtkreis der Glaslaternen dämmert eine Stadt, die es nicht mehr gibt.

Gründerzeitfassaden, gealterter Jugendstil, Sachlichkeit, die einmal neu war.

Harm: Mein Vater war Lehrer. Wir lebten in einer geräumigen Altbauwohnung. Hohe Decken, Stuck in einigen Räumen. Alles sehr hell wegen großer Fenster und weißer Decken. Oftmals habe ich auf dem Boden im Wohnzimmer gelegen und einfach nur die Ornamente an der Decke studiert.

Auf Sankt Nikolai liegt die Hoffnung begraben, mit bunten Blumen bepflanzt, gehegt und gepflegt. Die Bäume sind so alt, dass ihre Wurzeln die Särge umarmen.

Harm: Meine Eltern liegen zusammen mit meiner Schwester in einem Familiengrab. Auf der Sonnenseite des Friedhofs. Die Morgensonne scheint auf ihr Grab. Ich besuche sie einmal im Jahr. Dann lass' ich die Erinnerungen hochleben. Sehe meine Schwester noch einmal, wie sie morgens beim Frühstück in der Küche sitzt und in die Morgensonne blinzelt.

Mit der Gewissheit der Jahreszeiten, die Vogelzüge, mit der Geduld.

Laura: Ich hatte so viel Geduld, aber es war ein Unfall. Mein innerstes Gefühl wusste, dass er sterben würde, aber ich hatte immer noch Hoffnung gehabt. 
Ins Dickicht eines verwilderten Grabes gefallen der kupferne Engel ohne Kopf, sein linker Arm nur noch ein rostiges Eisen, in seiner Rechten fault Laub; im Herbst vor vier Jahren lächelte er noch.

Laura: Er war noch jung. Er war erst vier Jahre alt als er gestorben ist. Er hatte eine Zukunft, doch dann ist alles schlecht verlaufen. Es war ein Herbsttag, als er über die Strasse gelaufen ist. Er hat das Auto nicht gesehen.

Mütter gehen einkaufen, halten ihre sorgfältig aufgemalten Gesichter der Sonne entgegen und stopfen ein Brötchen ins Kindergeschrei.

Harm: Meine erste Freundin in Jugendtagen. Wir lagen gerne in der Sonne. Einen Sommer lang waren wir verliebt. Typische Jugendliebe. Ich kann mich so manches Mal noch genau an den Geruch erinnern - der Geruch ihres Gesichtes, sonnenbeschienene Haut gemischt mit dem Geruch von Sonnencreme und darüber die Frische der hereinwehenden Seeluft.

Estella: Bei mir zu Hause, halten die Mütter ihre schnell gereiften, von den Spuren des Lebens gekennzeichneten Gesichter, der Sonne entgegen und versuchen so, für einen Moment ihre Armut zu vergessen. Auch wenn sie der Gedanke nicht loslässt, wie sie in den nächsten Monaten den Hunger ihrer Kinder stillen könnten.

Ihr Nachwuchs klammert sich ängstlich an dieses Manna des Schweigens. Es ist so still hier. An der Steilküste des Traums, verschrien von Möwen und Kormoranen, von den Fischen verschwiegen, die an Land gehen im Netz. In den Fassaden haust Geschichte, unbereinigt, wuchernd gegen die Zeit.

Hinter den Tapeten Neuigkeiten von 1956, von 1942, 1930 und früher.

Irene: Alte Tapeten habe ich bestimmt, aber nie klebe ich ein Stück Tapete über eine andere. Ich tapeziere nur saubere Wände, weil meine Mutti und ihre Mutti und alle Frauen meiner Familie sehr stolz auf unsere sauberen Häuser sind.

Unter Zwischendecken versteckt Ölanstriche und Stuckornamente, tote Räume über den Lebenden, zwischen ihnen.

Laura: Nach der Beerdigung meines Sohnes fühlte ich mich deprimiert. Ich hatte vier Jahre immer wieder darüber nachgedacht. Wegen mir ist er geboren. Wegen mir ist er tot. Jedes Ornament haftet in meinem Gedächtnis. Alles ist eine Erinnerung. Unter der Erde gibt es nur tote Räume und darüber die Lebenden.

Die meisten Zimmer scheinen zu groß für den alltäglichen Gebrauch, [...]

Harm: Als ich in meine jetzige Wohnung zog, ich habe bewusst eine kleine ausgewählt, hatte ich quasi keine Einrichtung. Ein Tisch in der Küche, ein Schreibtisch, ein Bett. Eine handvoll Bücher, Besteck und Geschirr 
für eine Person, ein Laptop auf dem Schreibtisch. Kein Telefon. Wollte, musste allein sein. Die Wohnung schien riesig, ohne Leben. Es dauerte, sie zu füllen.

[...] ihre Bewohner fühlen sich benachteiligt und achten auf das pünktliche Erledigen der Hausordnung.

Karl: Mein Dienstmädchen kommt jeden Tag um 9 um die Wohnung zu putzen. Sie macht mein Bett, bringt meine Kleidung zur chemischen Reinigung, und hat jeden Tag um ein Uhr mein Mittagessen auf dem Tisch.

Manchmal glaube ich, das Meer ist nur eine Legende. Hinter den Häusern sind große Höfe verborgen mit Kirschbäumen, Flieder, Himbeerhecken und mit Birken, die über die Dächer ragen. Farbige Balkone lehnen sich in ihr Grün, zeigen Flagge: Handtücher und Laken.

Harm: Zumindest fühlte ich mich so, als die Aufregung des Eingewöhnens vorüber war. Da war wieder alles, wovor ich fliehen wollte. Das Meer, das mir die große Freiheit versprochen hatte, war wie verschwunden, als hätte sich die Welt geöffnet und das Meer verschluckt. Als wäre ich niemals weggegangen und immer noch dort. Vor meiner Abreise.

Estella: Bei uns gibt es keine Kirschbäume, Flieder oder Birken, auch keine farbigen Balkone. Aber Handtücher und Laken hängen an einer alten Kordel. Gehalten von zwei alten Holzstämmen, flattern sie im Wind und um sie herum laufen spielende Kinder, die nichts besitzen und dennoch glücklich sind.

Harm: Meine Großeltern hatten ein Haus mit Hof. Sie hatten Obstbäume und auch Kartoffeln in ihrem Hof angebaut. Einmal im Jahr waren alle zur Kartoffelernte dort. Doch das Größte war im Sommer, in die Bäume zu steigen und Äpfel zu pflücken. Nichts schmeckt so gut, wie ein eben gerade selbst gepflückter Apfel.

An Sommerabenden fallen mit spitzen Schreien die Mauersegler ins Geviert. Und die Nacht macht alles deutlicher: das Kreischen der Frau gegenüber, das ferne Johlen eines Betrunkenen, [...]

Karl: Dieser Satz erinnert mich an die großen Feiern mit meiner Mannschaft als wir vor sechs Jahren zum ersten Mal Jahren die Premier League gewonnen haben. Die ganze Mannschaft ist nach dem Spiel in Manchester ausgegangen und es war eine unglaubliche Nacht. Ich war erst achtzehn Jahre alt und hatte nur ein paar Spiele mit der ersten Mannschaft gespielt, aber in dieser Nacht waren wir alle Meister und Helden und ich habe mich zum ersten Mal wie ein Erwachsener gefühlt. Ich erinnere mich noch genau an das Gefühl allgemeiner Freude, als wir die Trophäe durch die Straßen getragen und präsentiert haben.

[...] die schweren Träume, das langsame, kaum merkliche Atmen der Häuser und weit weg ein Summen und leises Klirren, das zum Rest der 
Stadt gehören muss. Früher war da noch dieser hohe, singende Ton, wenn die letzte gelbe Acht gegen dreiviertel zwei durch die Kurven der Kaßbergauffahrt quietschte. Die Gaslaternen hellen nichts auf, aber man kann sich an ihnen orientieren, wie an den wenigen spät noch erleuchteten Fenstern.

Ein alter Mann in einer Dachwohnung, der Nacht für Nacht Bücher übersetzt.

Sabine: JedeNacht, bevorich einschlafe, mussich ein Buch lesen. Manchmal auf Englisch und manchmal auf Deutsch. Englisch ist meine Muttersprache, deshalb schlage ich manchmal ein paar Wörter im Wörterbuch nach.

Jack: Mein Vater spricht nicht mehr so viel. Er sieht immer traurig aus und macht nichts außer Lesen und Schlafen. Jeden Tag liest er eine Zeitung, isst sein Abendessen und geht mit einem Buch ins Bett. Ich finde das langweilig und kann nicht gut sein!

Eine Malerin, die sich eine Kindheit, einen Rausch erfindet.

Irene: Ich kann mich jeden Tag an meine Kindheit erinnern - ich muss die nicht erfinden - aber ich genieße die Kindheit meiner Großenkel mehr als meine eigene Kindheit, denn die können heutzutage viel mehr machen. Am Sportunterricht können die Mädchen jetzt teilnehmen, und die Kinder können andere Städte mit einem Chor, einer Band, usw. besuchen.

Ein trauriges Gesicht hinter der Scheibe im ersten Stock.

Laura: Das Gesicht war meins. Das Gesicht einer bestürzten Mutter im ersten Stock des großen Wohnhauses. Ich versuchte mir zu verzeihen und sah aus dem Fenster.

Ausschau haltend nach den Gespensterschiffen der Freibeuter, nach dem Auftauchen des Vergessenen, nach Wörtern, die Wasser aus den Mündern spült von Matrosen, Soldaten, ertrunkenen Mädchen, nach dem Verschwinden des Horizonts. Manchmal scheint es, als ob der Berg sein eigenes Gegenwort birgt: eine Höhle wäre, außer der Zeit. Und nur hier komme ich auf den Gedanken, dass das, was unter den Gullydeckeln rauscht, ja das Meer sein könnte.

Irene: Ich erinnere mich ganz klar daran - es war das erste Mal, dass ich das Meer sah. Als ich ein Kind war, wohnte meine Familie in Maine, aber nicht direkt am Strand, sondern zwei Stunden mit dem Auto entfernt. Ich dürfte erst acht Jahre alt gewesen sein, und meine ganze Familie, vier Kinder und meine Eltern, stiegen ins Auto ein. Als wir den Strand erreichten, standen meine Eltern da und umarmten sich. Das war 1942. 


\section{F Anhang $F$}

\section{F.1 Cork Radio}

(Laura (Moderatorin) sitzt an ihrem Moderatorentisch)

Laura: Radio Cork, Live Sendung! Guten Abend. Wir senden heute, am Abend des 29sten März, in Erinnerung an die erste Radio Cork Sendung 1927, hier vom renovierten Corker Frauengefängnis. Heute darf ich vier Gäste herzlich willkommen heißen. Ganz nach dem Wunsch unseres damaligen Post- und Telegrafenministers, Herrn J.J Walsh, halten wir die heutige Sendung sehr traditionell. Wir geben Ihnen einen Überblick, was in unserem schönen Cork so passiert ist. Wussten Sie zum Beispiel schon, dass die Königin Elizabeth, die Erste von England, am 24. März 1603 gestorben ist. James der Erste wurde König und er machte den Weg für die Religionsfreiheit der Katholiken frei. Sie konnten sich jetzt öffentlich zu ihrer Religion bekennen und es war erlaubt, in die Kirche zu gehen. Was halten Sie von Religion, Sabine?

Sabine: Ich bin katholisch. Jeden Sonntag feiern wir Messe, wo meine ganze Familie zusammen hingeht. Als ich jünger war, habe ich immer den Begriff „Beerdigung“ verwirrend gefunden. Er war kompliziert für mich zu verstehen. Warum gab es einen Sarg? Warum beten wir zu jemandem, den wir nie getroffen haben? Warum müssen wir in einem Grab begraben werden.

Laura: Religion hatte auch einen großen Stellenwert für Arbeiter, die in die Stadt Cork kamen. Sie gründeten die Young Men Society in Cork' und konnten so ihre Religion öffentlich ausüben. Estella, was ist Ihre Meinung dazu?

Estella: Auch in Brasilien hat Religion einen hohen Stellenwert. Neben den zwei großen Konfessionen haben sich unzählige Sekten gebildet, die angeben, im Namen Gottes zu handeln. Das Traurige jedoch ist, dass viele von diesen Sekten ihre Mitglieder abhängig machen und ihnen den letzten Pfennig aus der Tasche ziehen. Ich werde natürlich wütend, wenn ich in meinem Freundeskreis mitbekomme, wie blind die Menschen ihr Leben diesen Sekten opfern.

Irene: Ach! Radio ist so unaufdringlich und phantasievoll - man kann einfach zuhören und sich alles vorstellen. Ich finde das ganz gut, weil wir in einer visuellen und materialistischen Welt leben. Ich sehe meine Enkel - die sitzen mit glänzenden Augen vorm Fernseher - und ich hoffe, dass sie die Welt draußen nicht vergessen haben. Hören sie mal! Die Welt ist so schön.

Harm: Ein wirklicher Fortschritt für die Technik!

Laura: Auch elektrische Oberleitungen für Züge wurden hier bei uns in Cork eingeführt, meine Damen und Herren. Dieses revolutionäre System wurde am 22. Oktober 1889 vorgestellt. Es wurde von Bruder Dominic Burke von der North Monastry und Gerald Percival, einem Elektriker, entworfen. Ganz anders als das „Mittelschienensystem“, das in Großbritannien und Frankreich damals benutzt wurde... 
Harm: Wissen Sie, der Zug ist schon die angenehmste Art zu reisen. Man kann sitzen und lesen oder arbeiten. Ich bin schon immer gerne Zug gefahren. Es ist so schön einfach und simpel. Früher gab es Tickets, mit denen man einfach ein ganzes Wochenende durch die Gegend fahren konnte.

Irene: Und man muss nicht die ganze Zeit sitzen.

Laura: Aber auch medizinisch gibt es einiges aus Cork zu berichten ...

Jack: In seinem Buch, "The County and City of Cork Remembrancer", sagte Francis H. Tuckey, dass Pocken am 8. Februar 1818 weit verbreitet waren. Ich finde dieses Ereignis wichtig, weil es eine medizinische Leistung ist! Als ich jung war, war mein Vater in einen Verkehrsunfall verwickelt. Ich dachte, dass er tot wäre aber er hat überlebt. Die technologischen Entwicklungen in der Medizin sind sehr wichtig, weil mein Vater durch diese Entwicklungen gerettet wurde. In sechs Wochen im Jahr 1855 wurden ungefähr 1.000 Kinder durch Impfungen behandelt. Ohne diese medizinischen Maßnahmen wären diese Kinder vielleicht gestorben. Es ist klar zu sehen, dass in der Vergangenheit medizinische Entwicklungen sehr wichtig waren und auch heute noch sind.

Laura: Also, Grüß Gott Carl Fletcher, bekannter Fußballspieler bei Manchester United - es ist schön, Sie im Studio zu haben. Wir sprechen heute über die Geschichte der Stadt Cork und es interessiert uns Ihre Meinung dazu.

Carl: Danke, es ist mir immer ein Vergnügen, mit einem Fan zu sprechen.

Laura: Am zweiten August 1762 wurde das erste öffentliche und kostenlose Krankenhaus für Privatpersonen in Cork eröffnet. Vor dieser Eröffnung hatte die Regierung kein Krankenhaus für dieArmen der Stadt eingerichtet. Ein schrecklicher Gedanke.

Carl: Diese Situation erinnert mich nur allzu gut an mein eigenes Hilfswerk. An meinem vorherigen Wohnort London gibt es große Armut, und die medizinischen Einrichtungen waren unterentwickelt und das nächste Krankenhaus war eine halbe Stunde weg und immer überfüllt. Ich habe vor drei Jahren 500,000 € gespendet, um ein Kinderkrankenhaus dort zu bauen.

Laura: Was war Ihre Motivation für solche Wohltätigkeit?

Carl: Ich hatte persönliche Gründe dieses Krankenhaus bauen zu wollen. Mein bester Freund hatte sein Bein gebrochen, als wir jung waren. Wegen einer verspäteten Behandlung im nächsten Krankenhaus blieb sein Bein permanent geschädigt und er konnte nie wieder Fußball spielen. Er war ein besserer Spieler als ich und wir haben gedacht, dass er für große Dinge bestimmt wäre, aber das Schicksal hat sich eingemischt. Ich kann mich noch an die Tage erinnern, die wir mit Fußball spielen im Garten zugebracht haben. Er ist noch immer eine Inspiration für mich. (Carl weint)

Laura: Ein emotionale Geschichte Carl. Danke Carl. So, das wäre es für heute. Morgen geht es weiter mit neuen Gästen, neuen Geschichten und hoffentlich wieder mit Ihnen. Ich möchte mich bei meinen heutigen Gästen herzlich bedanken... Schönen Abend noch und lassen Sie sich 
noch ein paar wohlgemeinte Ratschläge für Ihren morgigen Tag geben. 